\title{
MAGELLANIC GLOBULARS AS COSMOLOGICAL TOOLS
}

\author{
ALVIO RENZINI \\ Astronomy Department \\ University of Bologna \\ CP 596 1-40100 Bologna \\ Italy
}

\begin{abstract}
The globular clusters (GC) of the Magellanic Clouds play a very important role for many astrophysical and cosmological topics. For example, they represent the ideal testground for stellar evolution theory, they allow us to study the the early dynamical evolution of star clusters, to obtain accurate initial mass functions in a fairly extended mass range, to calibrate the Cepheid period-luminosity relation, and so on. In this brief paper I will touch upon two items which are of considerable cosmological interest, and about which Magellanic Cloud globulars provide unique information. These topics concern i) $\mathrm{GC}$ formation in galaxies, and ii) the epoch of galaxy formation.
\end{abstract}

\section{The formation of globular clusters}

Several years ago Peebles and Dicke (1968) noticed that at the epoch of recombination the Jeans mass and the Jeans radius are respectively of the order of $\sim 10^{6} M_{\odot}$ and $\sim 10 \mathrm{pc}$, fairly close to the mass and radius of galactic globulars, and suggested that GCs might have been the first stellar systems to form out of the primordial matter, thus providing the building blocks for the later assembly of whole galaxies. However, this otherwise very elegant and attractive scenario has to face two main serious objections, which seem to me fatal for the primordial theory of GC formation.

The first problem is that Galactic GCs contain metals, i.e. their composition is far from being that of matter just emerged from the Big Bang. Not only so, but each cluster is extremely homogeneous, ${ }^{1}$ as revealed by the very tight red giant branch (RGB), which sets an upper limit of only a few per cent to the star-to-star metallicity dispersion in one cluster. The most straightforward interpretation of GC homogeneity is that metals pre-existed GC formation, and thus GCs are not primordial objects, but are rather LATE products in the EARLY organization of galaxies (Renzini 1983; Sandage and Tammann 1984). Indeed, GC self-enrichment of metals would require a very ad hoc formation process. Only massive stars should form at first, then they would have to evolve and eject their metals while still no low-mass star formation has started. Effective mixing should then homogenize the supernova ejecta with primordial matter, the large amount of energy injected by the massive stars should be efficiently dissipated, and only then should the low mass stars that we see today start forming. This does not look to be a physically realistic process. Besides that, concerning the required efficient mixing, a quantitative exploration of the idea reveals that it is hardly viable (Murray and Lin 1990).

\footnotetext{
${ }^{1}$ With the two notable exceptions of the GCs $\omega$ Cen and M22
} 
The second argument against the primordial nature of GCs is that the Magellanic Clouds still manage to form them, some 15 Gys after recombination. So, there must be other ways of making GCs, ways which do not appeal to very special primordial conditions, but are still working now before our eyes. Fall and Rees $(1985,1988)$ argue that during protogalactic collapse giant clouds with free fall time equal to the radiative cooling time are characterized by masses in the range between $\sim 10^{5}$ to $10^{6} M_{e}$, again, tantalizingly close to the mass range of Galactic GCs, and Fall and Rees suggest that this may be the key to understanding GC formation. If so, what about Magellanic Cloud globulars? We should conclude that either the protogalactic conditions were not so special, i.e. that they were not so different from today's Magellanic Cloud environment, or again that there have to be other ways of making GCs.

This kind of argument has been occasionally rejected by arguing that Magellanic Cloud globulars are different beasts. Are they really so different? The different beast argument relies on the consideration that the average mass of old Galactic GCs is $\sim 10^{5} M_{e}$, while that of young Magellanic GCs currently forming is only $\sim 10^{4} M_{\theta}$, i.e. they are one order of magnitude less massive beasts. However, I do not believe that this is a correct way of comparing the two cluster families. If one constructs a histogram of the age (not log age!!) distribution of Magellanic GCs perhaps the most striking aspect is that over one half of all clusters (i.e. of the objects listed in the catalog of van den Bergh 1981) are younger than $\sim 10^{8} \mathrm{yr}$. It seems that in the last $\sim 1 \%$ of their life the MCs have been able to produce as many clusters as in the previous $99 \%$. Are we witnessing a burst of GC formation? Almost certainly not! It is just a selection effect: currently forming GCs with $M<10^{4} M_{\Theta}$ are not able to stay in a magnitude limited catalog - such as that of van den Bergh - for more than $\sim 10^{8} \mathrm{yr}$. Due to their passive evolution they just fade away on nearly this timescale, and their integrated luminosity falls below the threshold for being included in a cluster catalog. Moreover, dynamical disruption may also assist this evaporation process.

But take the case of NGC 1866. This is a fairly respectable GC even according to Galactic standards, and will not soon drop off the catalog. This cluster has already an age of $\sim 10^{8} \mathrm{yr}$, with still $L \simeq 10^{6} L_{\odot}$ and $M \simeq 10^{5} M_{\odot}$. So, the LMC has been able to make at least one good GC in $10^{8} \mathrm{yr}$, i.e. in the last $1 \%$ of its lifetime. In van den Bergh's catalog there are roughly 100 clusters younger than $10^{8} \mathrm{yr}$, and therefore the average production rate has been of $\sim 1$ (small) cluster every million yr. If the formation time of a cluster is comparable to its free fall time (indeed, $\sim 10^{6} \mathrm{yr}$ ), then in the LMC there should be $\sim 1$ cluster in the making, and everybody will agree that 30 Doradus is just that cluster. However, the case of NGC 1866 seems to indicate that the LMC takes a nearly 100 times longer time interval to have a chance to make an average $\left(10^{5} M_{\odot}\right)$ cluster and on average it may take very many Gyr to produce a big (several $10^{5} M_{\odot}$ ) cluster. This is not, then, a special moment in MC history, and the age histogram of a magnitude limited sample of GCs did not look any different, e.g. $\sim 1$ Gyr ago, and will not look any different in a few Gyr from now. There is just a continuous replenishment of new small clusters as those already formed fade away, and only rarely a fairly massive cluster is formed which manages to remain brighter than the threshold for many Gyr.

From the above arguments, I tend to conclude that there is no compelling evidence for the present cluster productivity in the Magellanic Clouds (i.e. the production rate per unit gas mass) being any different from what it was in our own Galaxy some $15 \mathrm{Gyr}$ ago. I also do not see any compelling evidence against the possibility that even the GC mass function at formation could have been nearly the same, as certainly we should take into due consideration all the evolutionary processes which select the clusters able to survive for $15 \mathrm{Gyr}$ in the Galactic environment (cf. Fall and Rees 1977), as well as the fact that the protogalaxy had perhaps several hundred to one thousand times more gas out of which to make clusters than the LMC has today. 
What, then, are the implications for a comprehensive theory of cluster formation? What today does the LMC have in common with the protogalaxy that spiral disks do not have? It seems to me that plenty of gas and molecular clouds, coupled with a low density of angular momentum, may represent the necessary and sufficient ingredients for the formation of GCs. Strong shocks may also help, as usual, but it is not difficult to get them even in a small galaxy, without much internal motions. What remains to be understood is, of course, the cluster formation process itself, with its fragmentation into stars with a certain IMF and all that. But this is a very, very complicated hydrodynamical problem, which may escape our understanding for a fairly long time.

\section{The epoch of galaxy formation}

Very high redshift galaxies are now being discovered in what used to be the empty fields of 1 Jansky radiogalaxies, and objects at redshifts as high as 3.4 (Lilly 1988) and 3.8 (Chambers, Miley, and van Breugel 1990) have been reported. These galaxies are characterized by a "flat UV plus red bump" spectral energy distribution in the rest frame optical and near IR, and Lilly (1988) has proposed that the presence of the red bump requires a rather old age ( $>\sim 1 \mathrm{Gyr}$ ) for the bulk of the stars in the galaxy. At such a high redshift the universe itself is not much older than $\sim 1 \mathrm{Gyr}$, which inevitably pushes the epoch of galaxy formation back to extremely high redshifts, $z \sim 10$ 20. In turn, this is at variance with the standard cold dark matter scenario for the formation of galaxies and structures, which prefers galaxies to form rather later, at $z \sim 5$.

However, Lilly's determination of the age of the bulk stellar population of the high redshift galaxy relies on spectophotometric models (Bruzual 1983) which assume a $1 \mathrm{Gyr}$ duration of the star formation process, and in which the red giant component is not adequately treated. This may have affected the age determination, perhaps leading to a major overestimate of the real age. More recently, Chambers and Charlot (1990) have re-analyzed the problem, concluding that even the highest redshift galaxy in their sample (with $z=3.8$ ) has an age of only $\sim 3.3 \times 10^{8} \mathrm{yr}$, thus somehow relieving the pressure on the dark matter scenario. What is new in Chambers and Charlot's approach compared to that of Lilly? First, they have assumed a shorter duration for the star formation process (i.e. 108 rather than $10^{9} \mathrm{yr}$ ) and this certainly helps to keep the derived age low. Second, and perhaps more fundamentally, they have attempted a somewhat more sophisticated approach to the population synthesis, in particular for the treatment of the red giant evolutionary phases, i.e. precisely those stars which must be responsible for the red bump.

The crucial question with the red bump can be formulated as follows: at what age does an evolving stellar population first turn red? From a theoretical point of view the reddening of an evolving population is related to the first appearance of bright asymptotic giant branch (AGB) stars, as soon as objects with a degenerate C-O core appear (Renzini 1981; Renzini and Buzzoni 1986). A further increase in strength of the red bump is predicted when the red giant branch (RGB) also fully develops later as a consequence of the appearance of stars with degenerate helium cores. The precise age at which these population phase transitions take place is, however, still rather uncertain. The age at the AGB phase transition is in fact sensitive to both the adopted mass loss on the AGB, and to what one is willing to assume about the size of convective cores (the popular question of convective overshoot). Seemingly, also, the age at the RGB phase transition is sensitive to the adopted amount of overshoot, and for the standard case (no overshoot at all) it is $\sim 6 \times 10^{8} \mathrm{yr}$, independent of composition (Sweigart, Greggio and Renzini 1989, 1990).

Given the uncertainty affecting stellar models for the age of both the AGB and RGB phase transitions, Chambers and Charlot (1990) - rather than relying on theory - construct semiempirical spectral energy distributions implanting Magellanic Cloud GC spectra on Bruzual's models, thus adopting Magellanic GCs as template stellar populations. This is a very wise approach to the 
problem, because Magellanic globulars are indeed our best tool in trying to answer the question above, i.e. the age at which evolving stellar populations first turn red. Chambers and Charlot assume the AGB and RGB phase transitions to take place respectively at $t=3 \times 10^{8}$ and $1.2 \mathrm{x}$ $10^{9} \mathrm{yr}$. These numbers are consistent with quite a substantial overshoot, but Chambers and Charlot do not provide much detail in support of these assumptions. Yet, the derived age of the youngest radiogalaxy turns out to be just 3.3. $x 10^{8} \mathrm{yr}$, i.e. only $10 \%$ more than the adopted age at the AGB phase transition. This illustrates at once both that the red bump has been (implicitly!) attributed to the AGB phase transition, and that the derived age of the galaxies is crucially dependent on precisely at what age a stellar population is supposed to experience its AGB phase transition, and therefore on the precise dating of the few pertinent Magellanic globulars. I wish to emphasise strongly that high $\mathrm{S} / \mathrm{N}$ spectra of high redshift radiogalaxies do not sufice by themselves to determine the epoch of galaxy formation. We also need an accurate and reliable dating of MC clusters, i.e. we also need a good clock.

The cluster NGC 1866 neither shows an extended RGB, nor contains bright AGB stars (Frogel, Mould and Blanco 1990), and therefore it is younger than the age at the AGB phase transition. It is a type III cluster according to the classification of Searle, Wilkinson and Bagnuolo (1980). On the other hand, type V clusters do show a well-developed RGB and contain bright AGB stars and one can conclude that both the AGB and the RGB phase transitions take place within the age range spanned by type IV clusters, i.e. within somewhat more than $\sim 10^{8} \mathrm{yr}$ to perhaps $\sim 2 \times 10^{9} \mathrm{yr}$. This is also confirmed by the large range in integrated $V-K$ colours spanned by type IV clusters, from $V-K \simeq 1$ up to $\sim 2.7$, and for what concerns the AGB by the direct estimate of its contribution to the integrated light of the clusters (Frogel, Mould and Blanco 1990).

Yet, the precise age at which the two phase transitions take place remains to be accurately determined. Already several years ago an observational and theoretical project was begun aimed at determining the two ages and assessing the effects of the two phase transitions on the spectral energy distribution of stellar populations. The ultimate aim of the project is explicitly that of providing the astrophysicist and the cosmologist with an accurate, calibrated clock for estimating the age of high redshift galaxies. To this end, a systematic optical and near-IR study of type IV (and IV-like) Magellanic GCs has been undertaken and colour-magnitude diagrams have been obtained from both optical and near-IR photometry (Buonanno et al. 1988, Zinnecker et al. 1990) In parallel with this observational project, fine grids of stellar evolutionary sequences have been constructed up to the tip of the RGB (Sweigart, Greggio and Renzini 1989, 1990; see also Greggio 1987), and are being extended up to the onset of the thermally pulsing regime on the AGB. All in all, this is a very laborious and extensive kind of work, which for each cluster requires accurate photometry, calibrations, estimates of the field contamination and completeness, cross identification of stars in optical and IR frames, evaluation of the AGB and RGB contributions to the integrated light in various bands and in bolometric luminosity, and finally the age determination. So, we still have only preliminary results, but we hope to complete this investigation in the near future.

One aspect of particular concem is the relative paucity of GCs in this most interesting range of ages. With perhaps just a dozen type IV-like clusters (the LMC and SMC together do not provide much more than that), spanning some $2 \mathrm{Gyr}$, it may be difficult to pinpoint the precise age of the two phase transitions with an accuracy any better than $\sim 2 \times 10^{9} / 12$ or $\sim 2 \times 10^{8} \mathrm{yr}$. Small sample size (within individual GCs) may also be a problem, specially for the AGB phase transition. Ultimately, it might be that we will have to live with this kind of uncertainty concerning the age at which stellar populations first turn red, primordial galaxies first develop a red bump, and therefore with the uncertainty in the redshift of the epoch of galaxy formation which immediately 
follows. So straightforward indeed is the connection between type IV Magellanic clusters and 1 Jansky high redshift radiogalaxies!

I am very grateful to the Anglo Australian Observatory and its whole staff for its kind hospitality and stimulating scientific environment in the period during which this brief paper was written.

\section{References}

Bruzual, A., G. (1983), Ap. J. 273, 105.

Buonanno, R., Corsi, C.E., Fusi Pecci, F., Greggio, L., Renzini, A., Sweigart, A.V. (1988), IAU Symp. 126, The Harlow-Shapley Symposium on Globular Cluster Systems in Galaxies,

J.E. Grindlay and A.G.D. Philip, (eds.) (Kluwer: Dordrecht) p. 555.

Chambers, K.C., Charlot, S. (1990), Ap. J. 348, L1.

Chambers, K.C., Miley, G.K., van Breugel, W. (1990), Ap. J. in press.

Fall, S.M., Rees, M.J. (1977), M.N.R.A.S. 181, 37P.

Fall, S.M., Rees, M.J. (1985), $A p . J$. 298, 18.

Fall, S.M., Rees, M.J. (1988), IAU Symp. 126, The Harlow-Shapley Symposium on Globular Cluster Systems in Galaxies, J.E. Grindlay and A.G.D. Philip, (eds.) (Kluwer: Dordrecht) p. 323.

Frogel, J.A., Mould, J.R., Blanco, V.M. (1990), Ap. J. 352, 96.

Greggio, L. (1987), Stellar Evolution and Dynamics in the Outer Halo of the Galaxy, M. Azzopardi and F. Matteucci (eds.) (ESO: Garching), p.453.

Lilly, S.J. (1988), Ap. J. 333, 161.

Murray, S.D., Lin, D.N.C. (1990), Ap. J. 357, 105.

Peebles, P.J.E., Dicke, R.H. (1968), Ap. J. 154, 891.

Renzini, A. (1981), Annales de Phys. 6, 87.

Renzini, A. (1983), Mem. S.A.It. 54, 335.

Renzini, A., Buzzoni, A. (1986), Spectral Evolution of Galaxies, C. Chiosi and A. Renzini (eds.) (Reidel: Dordrecht) (Astrophys. Sp. Sci. Lib. V. 122) p. 195.

Sandage, A., Tammann, G. (1984), Large Scale Structure of the Universe, Cosmology and Fundamental Physics, G. Setti and L. van Hove (eds.) (CERN: Geneva) p.127.

Searle, L., Wilkinson, A., Bagnuolo, W.G. (1980), Ap. J. 239, 803.

Sweigart, A.V., Greggio, L., Renzini, A. (1989), Ap. J. Suppl. 69, 911.

Sweigart, A.V., Greggio, L., Renzini, A. (1990), Ap. J. in press.

van den Bergh, S. (1981), Astr. Ap. Suppl. 46, 79.

Zinnecker, H., Ferraro, F., Fusi Pecci, F., Renzini, A., Buonanno, A., Corsi, C.E., Terndrup, D. (1990), this volume. 